\title{
Secondary attention deficit/hyperactivity disorder due to right basal ganglia injury: A case report
}

\author{
Mehmet Fatih Ceylan**, Ömer Faruk Akcab \\ ${ }^{a}$ Department of Child and Adolescent Psychiatry, Dr. Sami Ulus Children's Hospital, Ankara, Turkey \\ ${ }^{b}$ Department of Child and Adolescent Psychiatry, Medical Faculty, Necmettin Erbakan University, Konya, Turkey
}

\begin{tabular}{|c|c|}
\hline ARTICLE INFO & ABSTRACT \\
\hline Article History & Attention deficit/hyperactivity disorder (ADHD) is a frequent and commonly studied \\
\hline $16 / 03 / 2013$ & neuropsychiatric disorder in children and adolescents. The symptoms of ADHD include \\
\hline Accepted $\quad 16 / 04 / 2013$ & $\begin{array}{l}\text { inattention and/or hyperactivity and impulsivity. Diagnosis of ADHD requires a persistent } \\
\text { pattern of symptoms beginning before the age of } 7 \text { except for secondary ADHD. }\end{array}$ \\
\hline $\begin{array}{l}\text { * Correspondence to: } \\
\text { Mehmet Fatih Ceylan }\end{array}$ & $\begin{array}{l}\text { Secondary ADHD may occur as a consequence of childhood traumatic brain injury. A } \\
\text { patient with secondary ADHD as a result of right basal ganglia injury is presented in this } \\
\text { case report. }\end{array}$ \\
\hline
\end{tabular}

Department of Child and Adolescent

Psychiatry,

J. Exp. Clin. Med., 2013; 30:189-191

Dr. Sami Ulus Children's Hospital,

Ankara, Turkey

e-mail: mehmetfceylan@yahoo.com

\section{Keywords:}

Basal ganglia

Closed head injury

Secondary ADHD

Traumatic brain injury

\section{Introduction}

Attention deficit hyperactivity disorder (ADHD) is a complex neuropsychiatric syndrome and also one of the most common childhood psychiatric disorders which affects between 5 and $10 \%$ of school-aged children (Scahill and Schwab-Stone, 2000).

Traumatic brain injury (TBI) is the most common neurological cause of death and disability in childhood. Cognitive and behavioral sequelae are common. Gerring et al. (1998) demonstrated that symptoms of ADHD develop in children after closed-head injury; these authors proposed that the diagnosis of secondary ADHD should be made when a child meets psychiatric criteria for ADHD (except possibly for age of onset), with symptoms developing after closed-head injury.

Neuroimaging studies on ADHD are focused on two regions in particular; the prefrontal cortex and the striatum, especially the caudate nucleus (Vaidya et al., 2005). In this case report, we present a patient who had a right basal ganglia injury after a closed head injury. His family applied to the hospital with complaints of inattentiveness, concentration problems and overactivity which occured after the trauma. We will discuss the etiology of secondary ADHD and its relationship with trauma on the basis of this case.

\section{Case}

The patient is a 10 years old boy who was brought to the child psychiatry clinic by his father with complaints of inattentiveness and overactivity. These complaints emerged following an accident that occured one year ago. He had fallen down while running and had a head trauma. He was unconscious for two days after the trauma and persistent myoclonic convulsive seizures localized in right side were observed. Diazepam was administered several times to control seizures, however, because this treatment failed, phenitoin was administered. In the first cranial MRI which was obtained just after the accident a hyperdense lesion was determined which impressed the right ventricle. Five days after the accident, computed tomography (CT), cranial CT angiography and electroencephalography (EEG) were applied to the patient in a university hospital. 
Cranial CT indicated decreased density in right basal ganglia due to previous infarction $(3 \times 3.5 \mathrm{~cm}$ in size and non-hemorrhagic) and loss of corticomedullar differentiation in right frontal area which suggested a possible previous infarction. Cranial CT angiography indicated a decrease of calibration in petrous, cavernous and subarachnoid segments of distal internal carotid artery which suggested dissection. EEG indicated no abnormality. The patient was referred to child neurology clinic and weakness in left upper (3/5) and lower (4/5) extremities were determined in his neurologic examination. As a conclusion of all clinical assessment, he was stated to have a subacute ischemia in his basal ganglia due to a dissection. Acetylsalicylic acid treatment of $2 \mathrm{mg} / \mathrm{kg} / \mathrm{d}$ and physiotherapy was administered to the patient. Six months after the accident, the patient had become more active and quickly got bored at school and home while studying and listening his lessons. His teacher had complaints of overactivity, irritability, difficulty in maintaining concentration and unwillingness to listening and studying lessons, after the accident.

In developmental history, he was born in a low sociocultural family environment as a single child of his family. He was born with a normal spontaneous vaginal delivery at term and his birth weight was $3000 \mathrm{~g}$. His motor and language development were in normal range. He had no a chronic illness including any psychiatric disorder or ADHD symptoms before the head trauma. In family history his mother was a 30 years old housewife and his father was a 50 years old farmer. They were divorced 7 years ago and the patient was brought up by his grandfather since then. There was no history of psychiatric disorder or ADHD in his family.

Psychiatric examination and psychiatric tests (K-SADSPL, Conner's parent and teacher rating scales) were administered to the patient. Because he met the diagnostic criteria of ADHD -except age of onset-, he was diagnosed as secondary ADHD. He described some depressive symptoms, despite he did not meet entire criteria for depression. He was administered medication of imipramin $35 \mathrm{mg} / \mathrm{d}$ and his ADHD and depressive symptoms relieved significantly.

\section{Discussion}

Brain disorders may also have a psychopathological impact on the capacity for emotional and cognitive coping and mastery. Associations between brain disorders and behavior may be direct; for example, brain systems involved in attention, emotion regulation and inhibition are dysfunctional in children with traumatic brain injury. In brain disorders, problems with inhibitory brain processes that are important in task mas- tery are common and may be linked to inattention and hyperactivity (Floden and Stuss, 2006). Aron and Poldrack (2005) examined the existent literature with a narrower focus on the neural correlations of inhibitory control rather than the broader construct of executive processes. Many studies support the hypothesis that dysfunctions in prefrontal cortex, basal ganglia, and the related neurotransmitter systems (dopaminergic, noradrenergic, and serotonergic) underlie inhibitory deficits in ADHD (Dickstein et al., 2006).

The basal ganglia, a subcortical group of gray matter nuclei, appear to mediate postural tone. The four functionally distinct ganglia are the striatum, the pallidum, the substantia nigra, and the subthalamic nucleus. Collectively known as the corpus striatum, the caudate and putamen harbor components of both motor and association systems. The caudate nucleus plays an important role in the modulation of motor acts (Vaidya et al., 2005). Anatomic studies of children with developmental ADHD demonstrate loss of volume of frontal and striatal structures but no lesions; thus ADHD may be manifestation of any injury to the striatum, as opposed to a developmental disorder. Children who develop secondary ADHD tent to have higher lesion fractions in the right putamen than children who do not develop secondary ADHD. Results for the right basal ganglia are suggestive in assocation with secondary ADHD (Herskovits et al., 1999). Similarly, right basal ganglia injury caused ADHD symptoms in the present case.

To our knowledge, this is the first report from Turkey that closed head injury caused secondary ADHD. Closed head injury do not cause only neurological problems. It may also conclude some psychiatric problems including cognitive and behavioral sequelae. Sometimes, behavioral sequelae may overlap with cognitive sequelae (Michaud et al., 1993). The most common acute psychiatric diagnosis is delirium with agitation, loss of orientation and limited attention span. In the chronic phase, post-traumatic change in personality can be observed like frontal lobe syndrome. Frontal lobe syndrome includes disinhibition, lack of judgment or foresight, and mood changes which can range from apathy to euphoria (Gerring et al., 1998). Social disinhibition, poor impulse control, hyperactivity, attention deficits, disinhibited talk and forgetfulness are also among the chronic sequelae of the closed head injury (Kaitaro et al., 1995).

As a conclusion, Psychiatry and Child Psychiatry specialists should consider behavioral changes followed by closed head injury.

\section{REFERENCES}

Aron, A.R., Poldrack, R.A., 2005. The cognitive neuroscience of response inhibition: Relevance for genetic research in attention-deficit/hyperactivity disorder. Biol. Psychiatry. 57, 1285-1292.

Dickstein, S.G., Bannon, K., Castellanos, F.X., Milham, M.P., 2006. The neural correlates of attention deficit hyperactivity disorder: An ALE meta-analysis. J. Child Psychol. Psychiatry. 47, 1051-1062.

Floden, D., Stuss, D.T., 2006. Inhibitory control is slowed in patients with right superior medial frontal damage. J. Cogn. Neurosci. 18, 18431849.

Gerring, J.P., Brady, K.D., Chen, A., Vasa, R., Grados, M., Bandeen-Roche, K.J., Bryan, R.N., Denckla, M.B., 1998. Premorbid prevalence of ADHD and development of secondary ADHD after closed head injury. J. Am. Acad. Child Adolesc. Psychiatry. 37, 647-654.

Herskovits, E.H., Megalooikonomou, V., Davatzikos, C., Chen, A., Bryan, R.N., Gerring, J.P., 1999. Is the spatial distribution of brain lesions associated with closed-head injury predictive of subsequent development of attention-deficit/hyperactivity disorder? Analysis with brainimage database. Radiology. 213, 389-394.

Kaitaro, T., Koskinen, S., Kaipio, M.L., 1995. Neuropsychological problems in everyday life: A 5-year follow-up study of young severely closed-head-injured patients. Brain Inj. 9, 713-727. 
Michaud, L.J., Rivara, F.P., Jaffe, K.M., Fay, G., Dailey, J.L., 1993. Traumatic brain injury as a risk factor for behavioral disorders in children. Arch. Phys. Med. Rehabil. 74, 368-375.

Scahill, L., Schwab-Stone M., 2000. Epidemiology of ADHD in school-age children. Child Adolesc. Psychiatr. Clin. N. Am. 9, $541-555$.

Vaidya, C.J., Bunge, S.A., Dudukovic, N.M., Zalecki, C.A., Elliott, G.R., Gabrieli, J.D., 2005. Altered neural substrates of cognitive control in childhood ADHD: Evidence from functional magnetic resonance imaging. Am. J. Psychiatry. 162, 1605-1613. 